\title{
UTILITY OF KIRBY BAUER DISC DIFFUSION METHOD FOR VANCOMYCIN SUSCEPTIBILITY TESTING OF STAPHYLOCOCCAL ISOLATES: PRACTICABILITY, ACCEPTABILITY, AND QUALITY ASPECTS.
}

Nandan T. M, Vidyasagar K, Ravikumar R.

\author{
1. Non PG Senior Resident, Department of Neuromicrobiology, NIMHANS, Bangalore. \\ 2. Non PG Senior Resident, Department of Neuromicrobiology, NIMHANS, Bangalore. \\ 3. Professor \& Head, Department of Neuromicrobiology, NIMHANS, Bangalore.
}

\section{CORRESPONDING AUTHOR}

Dr. R. Ravi Kumar MD,

Professor and Head,

Department of Neuromicrobiology, NIMHANS, Bangalore,

E-mail: ravikumarbly@yahoo.co.uk

Ph: +919448073965.

ABSTRACT: Testing MIC of Vancomycin for all staphylococcal isolates is mandatory according to the current CLSI guidelines and this will considerably increase the cost of culture and sensitivity testing. This study is an attempt to re-consider the utility of the conventional disc diffusion method for cost-effective testing in resource poor settings. MATERIALS AND METHODS: Thirty coagulase positive and twenty eight coagulase negative staphylococci from various clinical samples have been randomly tested for minimum inhibitory concentration along with the Kirby Bauer disc diffusion method for Vancomycin over a period of five months.

RESULTS: Susceptibility results of all the 58 isolates tested have been identical by both disc diffusion and HiComb MIC methods. Out of 58 isolates, 57 (98.26\%) staphylococci have been sensitive to vancomycin by Kirby Bauer disc diffusion method as well as by the MIC testing method. The MICs of the susceptible strains have been $<2 \mu \mathrm{g} / \mathrm{mL}$. One isolate, a coagulase negative staphylococcus, has been tested to be resistant to vancomycin by both the methods with an MIC of $32 \mu \mathrm{g} / \mathrm{mL}$ SUMMARY: In our study of staphylococcal isolates from various clinical samples, there is overt significant concordance between disc diffusion and MIC testing methods in the routine susceptibility testing of vancomycin. CONCLUSION: Kirby Bauer disc diffusion method may still be of utility for routine testing of vancomycin susceptibility except for few cases especially in resource poor settings.

KEYWORDS: Staphylococci, Vancomycin, disc diffusion, minimum inhibitory concentration, quality control.

INTRODUCTION: Updating Clinical Laboratory Standards Institute (CLSI) zone diameter interpretive standards for vancomycin susceptibility testing of any staphylococcal isolate no longer recommends disc diffusion methods. ${ }^{1}$ Testing MIC for all staphylococcal isolates is mandatory according to the current CLSI guidelines right from Jan 2010 and this will considerably increase the cost and time of culture and sensitivity testing procedures. This study is an attempt to reconsider the utility of the disc diffusion method for cost-effective testing methods in resource poor settings. This study is also an attempt to stress the need for framing indigenous performance and interpretive standards for antimicrobial susceptibility testing for validation of quality control criteria. The study has been performed in a tertiary care hospital in Bangalore, for a sample of randomly selected isolates between June and October 2011 over a period of 5 months on coagulase positive and coagulase negative staphylococci. 
MATERIALS \& METHODS: Randomly chosen 30 coagulase positive and 28 coagulase negative staphylococci isolated from various clinical samples have been tested by the Kirby Bauer disc diffusion method along with HiComb MIC test for minimum inhibitory concentration for Vancomycin by standard methods and the results compared and evaluated. Previous ${ }^{2}$ CLSI guidelines for disc diffusion zone diameter interpretive standards has been used for the disc diffusion method and the current ${ }^{3}$ minimum inhibitory concentration interpretive standards for the MIC method. Zone diameters $\geq 15 \mathrm{~mm}$ has been taken as susceptible to vancomycin for the disc diffusion method and, the MICs $\leq 2 \mu \mathrm{g} / \mathrm{mL}$ as "susceptible," 4-8 $\mu \mathrm{g} / \mathrm{mL}$ as "intermediate," and $\geq 16 \mu \mathrm{g} / \mathrm{mL}$ as "resistant" have been taken for the MIC method. All the plates were incubated for the complete $24 \mathrm{hr}$ period, carefully checked for heteroresistant subpopulation. Media, Vancomycin $(30 \mu \mathrm{g})$ discs and HiComb MIC strips for vancomycin were procured from HiMedia, India.

RESULTS: Susceptibility results of all the 58 isolates tested have been identical by both disc diffusion and HiComb MIC methods. Out of 58 isolates, 57 (98.26\%) staphylococci have been sensitive to vancomycin by Kirby Bauer disc diffusion method as well as by the MIC testing method. The MICs of the susceptible strains have been $<2 \mu \mathrm{g} / \mathrm{mL}$. One isolate, a coagulase negative staphylococcus, has been tested to be resistant to vancomycin by both the methods with an MIC of $32 \mu \mathrm{g} / \mathrm{mL}$. Table 1, lists the randomly selected sample types and the number of isolates. Table 2, lists the vancomycin zone diameter ${ }^{8}$ and MIC means and ranges. Table 3, lists the distribution of Staphylococci in the present study. Figure 1, shows the vancomycin Kirby Bauer disc diffusion test. Figure 2, shows the vancomycin HiComb MIC test. Fig 3: Age wise Distribution of male and female patients.

DISCUSSION: In our study of staphylococcal isolates from various clinical samples, there is significant concordance between disc diffusion and MIC testing methods in the susceptibility testing of vancomycin. The MICs tested for vancomycin in a study done in Southern India were $<2 \mu \mathrm{g} / \mathrm{ml} .^{4}$ Kirby-Bauer disc diffusion method for vancomycin may continue to be of routine practical utility in developing countries like India. Although vancomycin agar screen method is recommended for screening for less susceptible isolates, studies show in house vancomycin screen agar preparations are inferior to commercially prepared ones. ${ }^{5}$ Moreover, total prevalence of hVISA according to a meta-analysis supported by National Institute of Health, USA was $<2 \%{ }^{6}$ and studies from India are sparse. Different countries have been reporting variable incidence of hVISA attributable to variable MIC interpretation breakpoint criteria. ${ }^{7}$ So routine testing of all staphylococci for vancomycin by methods other than simple disc diffusion should not be made mandatory, in view of the increasing demand for National Accreditation Board for Testing and Calibration Laboratories (NABL) accreditation of lab testing, quoting CLSI as the performance and interpretive standards criteria for quality assurance in our country. In keeping the costs low for resource poor labs, any cost increases in routine testing practices will have negative impact on quality control practices and general acceptability of the testing itself. This also opens up a new arena where regulatory indigenous diagnostic interpretive standards for antibiotic sensitivity testing at the national level are framed in the Indian context with CLSI or any other international guideline as a possible model.

CONCLUSION: Kirby Bauer disc diffusion method may still be of utility for routine testing of vancomycin susceptibility except for few cases and there is an urgent need for national level 
guidelines pertaining to antimicrobial susceptibility reporting standards in clinical microbiology labs for standard practice and sustainable development of clinical microbiology in India.

\section{REFERENCES :}

1. Clinical and Laboratory Standards Institute. Performance standards for antimicrobial susceptibility testing; twentieth informational supplement. CLSI document M100-S18. Wayne, PA: Clinical and Laboratory Standards Institute; 2010.

2. Clinical and Laboratory Standards Institute. Performance standards for antimicrobial susceptibility testing; sixteenth informational supplement. CLSI document M100-S18. Wayne, PA: Clinical and Laboratory Standards Institute; 2006.

3. Clinical and Laboratory Standards Institute. Performance standards for antimicrobial susceptibility testing; twenty-first informational supplement. CLSI document M100-S18. Wayne, PA: Clinical and Laboratory Standards Institute; 2011.

4. Tenover, F. C., M. V. Lancaster, B. C. Hill, C. D. Steward, S. A. Stocker, G. A. Hancock, C.M. O'Hara, S. K. McAllister, N. C. Clark, and K. Hiramatsu. Characterization of staphylococci with reduced susceptibilities to vancomycin and other glycopeptides. J. Clin. Microbiol. 1998, 36:1020-27.

5. Liu, C., and H. F. Chambers. Staphylococcus aureus with heterogeneous resistance to vancomycin: epidemiology, clinical significance, and critical assessment of diagnostic methods. Antimicrob. Agents Chemother. 2003, 47:3040-45.

6. Bhateja P, Mathur T, Pandya M, Fatma T, Rattan A. Detection of vancomycin resistant Staphylococcus aureus: A comparative study of three different phenotypic screening methods. Indian J Med Microbiol 2005; 23:52-55.

7. G. A. Menezes, B. N. Harish, S. Sujatha, K, Vinothini ,S. C. Parija. Emergence of vancomycin-intermediate Staphylococcus species in southern India. Journal of Medical Microbiology. 2008, 57(7): 911-12.

8. D Oosthuizen, TR Walsh, GF Weldhagen. Detection of Staphylococcus aureus with reduced susceptibility to vancomycin in a South African teaching hospital. The South African journal of Epidemiology and Infection. 2005, 20 (4): 127-29

Table 1: Sample wise distribution of isolates

\begin{tabular}{|l|l|}
\hline Randomly Selected Sample Types & $\begin{array}{l}\text { No. of Isolates } \\
\text { from Various } \\
\text { Samples }\end{array}$ \\
\hline Abscess Wall & 1 \\
\hline Blood & 5 \\
\hline Brain Abscess Pus & 1 \\
\hline CSF & 12 \\
\hline Cystic Fluid & 1 \\
\hline Ear Swab & 1 \\
\hline EVD Tip & 2 \\
\hline Graft Site Swab & 1 \\
\hline Periorbital Pus & 1 \\
\hline
\end{tabular}

Journal of Evolution of Medical and Dental Sciences/Volume1/Issue5/November-2012Page-766 
BRIEF COMMUNICATION

\begin{tabular}{|l|l|}
\hline Pleural Fluid & 1 \\
\hline Pus Swab & 8 \\
\hline Shunt Tip & 2 \\
\hline Tracheal & 7 \\
\hline Urine & 2 \\
\hline Wound Swab & 13 \\
\hline $\begin{array}{l}\text { Total No. of Isolates from Various } \\
\text { Samples }\end{array}$ & 58 \\
\hline
\end{tabular}

Table 2: Vancomycin zone diameter and MIC mean and range

\begin{tabular}{|l|l|l|}
\hline & Mean & Range \\
\hline $\begin{array}{l}\text { Disc Diffusion zone } \\
\text { diameter in mm }\end{array}$ & 20.41304 & $7.0-32.0$ \\
\hline MIC in $\mu \mathrm{g} / \mathrm{mL}$ & 0.678902 & $0.016-32$ \\
\hline
\end{tabular}

Table 3: Distribution of staphylococci.

\begin{tabular}{|l|l|}
\hline Isolates & Number \\
\hline MSSA & 6 \\
\hline MRSA & 24 \\
\hline CONS & 10 \\
\hline MRCONS & 18 \\
\hline Total no. of Isolates & 58 \\
\hline
\end{tabular}

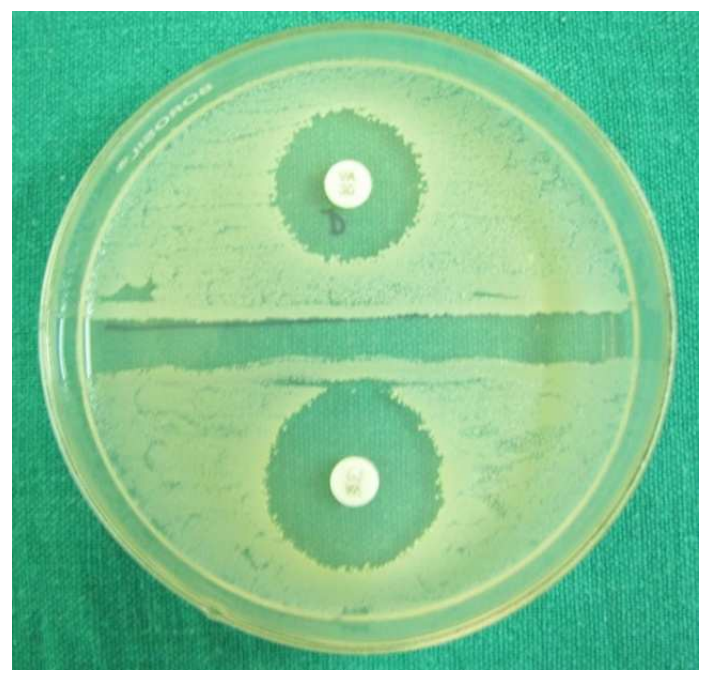

Fig. 1: Vancomycin testing by disc diffusion method 


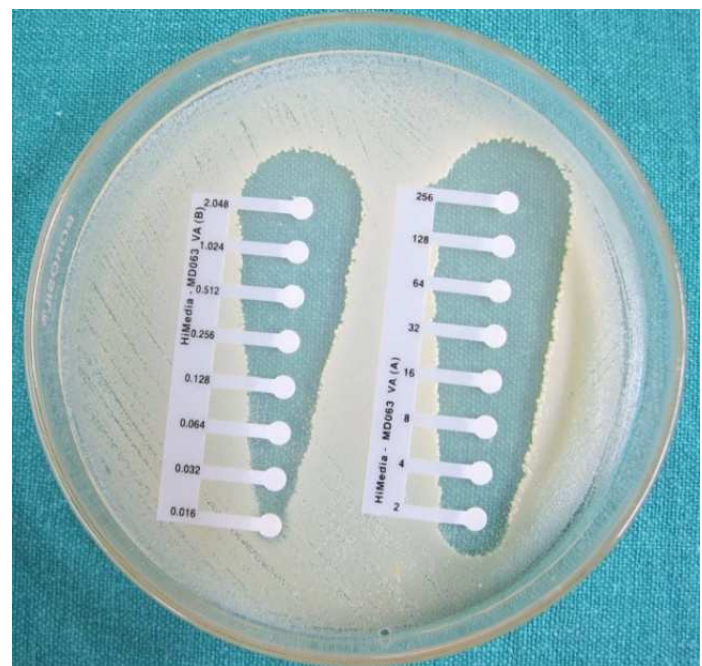

Fig. 2: Vancomycin MIC by HiComb method

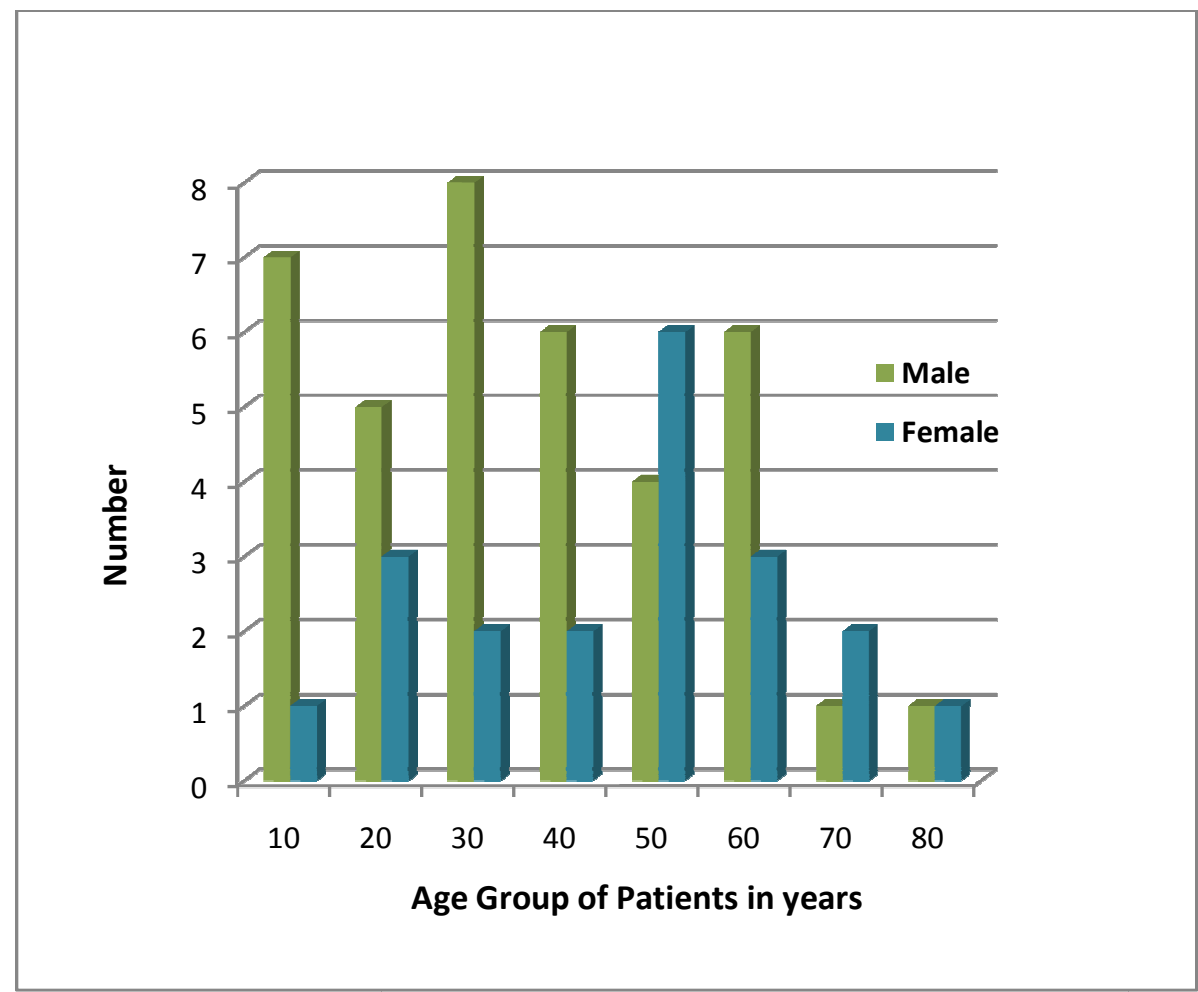

Fig 3: Age wise Distribution of male and female patients. 